\title{
Symptom Aggravation in Restless Legs Syndrome during Menstrual Cycle
}

\author{
Sungeun Hwang ${ }^{1}$, Yong-Won Shin ${ }^{2}$, Ki-Young Jung ${ }^{1,2}$ \\ ${ }^{1}$ Translational Medicine Major, Department of Medicine, College of Medicine, Seoul National University, Seoul, \\ ${ }^{2}$ Department of Neurology, Seoul National University Hospital, Seoul, Korea
}

Received December 9, 2019

Revised December 24, 2019

Accepted December 26, 2019

Address for correspondence

Ki-Young Jung, MD, PhD

Department of Neurology,

Seoul National University Hospital,

101 Daehak-ro, Jongno-gu,

Seoul 03080, Korea

Tel: +82-2-2072-4988

Fax: +82-2-2072-2474

E-mail: jungky10@gmail.com
Objectives: The purpose of this study is to confirm restless legs syndrome (RLS) symptom aggravation during menstrual period and verify factors related to symptom aggravation. Methods: A total of 20 premenopausal female RLS patients were classified into two groups according to symptom aggravation during menstrual period (menstrual RLS group and non-menstrual RLS group). They answered a questionnaire including duration and quantity of menstruation, other medical conditions, and premenstrual syndrome symptoms. Laboratory tests including iron panel and hemoglobin levels were done. Results: Six out of 20 patients (30\%) complained of symptom aggravation during menstrual period. RLS symptoms were aggravated by $40 \pm 33.47 \%$ compared to non-menstrual period in menstrual RLS group. One patient was taking additional medication for aggravated symptoms. Menstrual duration, quantity of menstrual bleeding showed no difference between menstrual RLS and non-menstrual RLS groups. On laboratory tests, two patients from non-menstrual RLS group were diagnosed with iron deficiency anemia. Serum iron levels, total iron binding capacity, serum iron saturation, and serum ferritin levels did not show difference between the two groups, while hemoglobin levels were significantly lower (13.8 vs. $12.4 \mathrm{~g} / \mathrm{dL}$ ) in non-menstrual RLS group $(p=0.044)$. Conclusions: RLS symptoms aggravate during menstrual period in $30 \%$ of premenopausal RLS patients. Low ferritin levels were not related to menstrual RLS symptom aggravation. Further study is required to verify other factors such as hormonal fluctuations.

J Sleep Med 2019;16(2):109-112

\section{Introduction}

Restless legs syndrome (RLS) is a common neurologic, sensorimotor disorder. RLS patients experience an urge to move the legs usually caused by unpleasant sensations, which is relieved by movement. ${ }^{1}$ Although pathophysiology of RLS is not fully discovered, dopamine and iron are considered to play substantial role in RLS pathophysiology. ${ }^{2}$ It is wellknown that lower serum ferritin correlates to greater RLS severity and iron supplementation can improve symptoms in RLS patients. ${ }^{3,4}$

RLS is more prevalent in women than in men, especially in pregnant women. ${ }^{5}$ In pregnancy, the prevalence of RLS is estimated to be $22 \%$ across all trimesters, gradually increasing until 7th to 8th month of gestation. Iron deficiency, which is

This is an Open Access article distributed under the terms of the Creative Commons Attribution Non-Commercial License (https://creativecommons.org/licenses/by-nc/4.0) which permits unrestricted non-commercial use, distribution, and reproduction in any medium, provided the original work is properly cited. common in pregnant women, is a well-known risk factor for developing RLS. Also elevated estrogen and progesterone levels are suggested to contribute in pathogenesis of RLS in pregnancy. ${ }^{6}$

Aggravating RLS symptoms during menstrual period in $29 \%$ of RLS patients have been reported. ${ }^{7}$ But there has been no study to identify factors correlated to RLS symptom aggravation during menstrual period. For menorrhagia can induce iron deficiency anemia (IDA) and women undergo hormonal fluctuations during menstrual cycle, iron deficiency and hormonal change may contribute to symptom aggravation during menstrual period.

The purpose of this study is to confirm the existence of RLS symptom aggravation during menstrual period in female RLS patients, and to identify factors related to symptom aggravation. 


\section{Methods}

Subjects were screened from female RLS patients who visited sleep clinics of Seoul National University Hospital (SNUH). Inclusion criteria were 1) premenopausal female, 2) 18 to 55 years old, and 3) diagnosed according to International Restless Legs Syndrome Study Group diagnostic criteria. Patients under anovulatory state with oral contraceptives, or patients with history of hysterectomy or bilateral oophorectomy were excluded.

The study was approved by Institutional Review Board (IRB, H-1612-080-814) of SNUH. Written informed consents were obtained from each subject.

Eligible subjects answered a questionnaire which included presence/absence of RLS symptom aggravation during menstrual period, relative degree of symptom aggravation, and necessity of additional dosage of RLS medication. Family history of RLS, duration of menstrual period, quantity of menstrual bleeding, symptoms related to premenstrual syndrome (anxiety, depressive mood, increased appetite, headache, fatigue, breast tenderness and abdominal bloating), augmentation, known history of IDA, diabetes mellitus (DM), and herniated intervertebral disc (HIVD) were included in the questionnaire. Degree of symptom aggravation was measured based on patients' recall as a percentage of perceived symptom aggravation compared to non-menstrual period. When symptoms were not changed during menstrual period, degree of symptom aggravation was described as $0 \%$. When a patient's symptoms were 2 times more severe in menstrual period, degree of symptom aggravation was described as $100 \%$. Quantity of menstrual bleeding was classified as 3 groups-less, moderate and much-based on each patient's perception. Laboratory test results including serum iron, total iron binding capacity (TIBC), iron saturation, ferritin and plasma hemoglobin were collected.

Subjects were divided into 2 groups-menstrual RLS group (patients with RLS symptom aggravation during menstrual period) and non-menstrual RLS group (patients without RLS symptom aggravation during menstrual period). Then characteristics and laboratory tests stated above were compared between 2 groups. For continuous variables, Shapiro-Wilk normality test was done first. If normality hypothesis was met, two sample t-test was used; otherwise, Wilcoxon Rank-Sum test was used. For categorical variables, Fisher's exact test or Cochran-Armitage trend test was used. R: A Language and Environment for Statistical Computing version 3.4.4 (R Foundation for Statistical Computing, Vienna, Austria) was used to conduct data analysis.

\section{Results}

A total of 20 premenopausal female RLS patients were enrolled. Six out of 20 patients (30\%) complained of RLS symptom aggravation during menstrual period. In menstrual RLS group, RLS symptoms were aggravated by $40 \pm 33.47 \%$ compared to non-menstrual period. Among 6 patients in menstrual RLS group, 1 patient complained of $100 \%$ symptom aggravation compared to non-menstrual period, and she needed additional dosage of medication to control aggravated symptoms. One patient from non-menstrual RLS group was taking oral iron supplement, who was diagnosed as IDA on laboratory tests, in spite of iron therapy.

Age, age at onset, disease duration, and incidence of family history showed no difference between the two groups (Table 1). Duration of menstrual period was $5.5 \pm 1.4$ days in menstrual RLS group and $6.0 \pm 1.6$ days in non-menstrual RLS group $(p=0.516)$. All 6 patients of menstrual RLS group replied to have moderate amound of menstrual bleeding, whereas 10 patients of non-menstrual RLS group (71.4\%) replied to have moderate amound of menstrual bleeding $(p=0.502)$. No patient from both groups had augmentation, known history of IDA, or DM. Only 1 patient from non-menstrual RLS group had HIVD. None of premenstrual syndrome symptoms showed statistically significant difference between the two groups (Table 2).

In laboratory tests, 2 patients from non-menstrual RLS group were diagnosed as IDA. No patient from menstrual RLS group showed anemia or iron deficiency. Serum iron, TIBC, iron saturation, and ferritin levels were not statistically different between the two groups (Table 3). Plasma hemoglobin was significantly lower in non-menstrual RLS group (12.4 $1.3 \mathrm{~g} / \mathrm{dL}$ vs. $13.8 \pm 1.2 \mathrm{~g} / \mathrm{dL}, p=0.044)$.

\section{Discussion}

This is the first study to investigate factors related to RLS symptom aggravation during menstrual period. In this study we confirmed that $30 \%$ of premenopausal female RLS patients experience symptom aggravation during menstrual cycle, as previously reported. ${ }^{7}$ As pregnant women undergo anemia and RLS symptom aggravation, we expected higher prevalence of anemia and iron deficiency in menstrual RLS group. In contrast, there was no significant difference in iron panel between the two groups and anemia was more frequent in non-menstrual RLS group, resulting in lower plasma hemoglobin levels. Also, there was no difference in menstrual duration and quantity of menstrual bleeding between the two groups.

One of possible explanation for this unexpected result is 
Table 1. Comparison of clinical characteristics between RLS patients with and without menstrual symptom aggravation

\begin{tabular}{lccc}
\hline \multicolumn{1}{c}{ Variables } & Menstrual RLS (n=6) & Non-menstrual RLS (n=14) & $p$ \\
\hline Age (years) & $29.5 \pm 6.2$ & $33.6 \pm 9.9$ & 0.368 \\
Age at onset (years) & $20.8 \pm 3.5$ & $24.9 \pm 10.8$ & 0.229 \\
Disease duration (years) & $7.5[4.0-11.0]$ & $6.0[2.0-11.0]$ & 0.590 \\
Augmentation & $0(0.0)$ & $0(0.0)$ & 1.000 \\
Family history & $4(66.7)$ & $6(42.9)$ & 0.629 \\
Menstrual duration (days) & $5.5 \pm 1.4$ & $6.0 \pm 1.6$ & 0.516 \\
Menstrual bleeding & & & 0.502 \\
$\quad$ Less & $0(0.0)$ & $1(7.1)$ & $10(71.4)$ \\
$\quad$ Moderate & $6(100.0)$ & $3(21.4)$ & 1.000 \\
$\quad$ Much & $0(0.0)$ & $0(0.0)$ & 1.000 \\
History of IDA & $0(0.0)$ & $0(0.0)$ & 1.000 \\
DM & $0(0.0)$ & $1(7.1)$ & \\
HIVD & $0(0.0)$ & \\
\hline
\end{tabular}

Data are presented as mean \pm standard deviation, median [interquartile range], or frequency (percentage). RLS: restless legs syndrome, IDA: iron deficiency anemia, DM: diabetes mellitus, HIVD: herniated intervertebral disc

Table 2. Comparison of premenstrual syndrome symptoms between RLS patients with and without menstrual symptom aggravation

\begin{tabular}{|c|c|c|c|}
\hline Variables & Menstrual RLS ( $\mathrm{n}=6)$ & Non-menstrual RLS ( $\mathrm{n}=14)$ & $p$ \\
\hline Anxiety & $2(33.3)$ & $4(28.6)$ & 1.000 \\
\hline Depression & $2(33.3)$ & $5(35.7)$ & 1.000 \\
\hline Increased appetite & $3(50.0)$ & $10(71.4)$ & 0.613 \\
\hline Headache & $2(33.3)$ & $3(21.4)$ & 0.613 \\
\hline Fatigue & $5(83.3)$ & $9(64.3)$ & 0.613 \\
\hline Breast tenderness & $4(66.7)$ & $6(42.9)$ & 0.629 \\
\hline Abdominal bloating & $5(83.3)$ & $10(71.4)$ & 1.000 \\
\hline
\end{tabular}

Data are presented as frequency (percentage). RLS: restless legs syndrome

Table 3. Comparison of laboratory variables between RLS patients with and without menstrual symptom aggravation

\begin{tabular}{lccc}
\hline \multicolumn{1}{c}{ Variables } & Menstrual RLS $(\mathrm{n}=6)$ & Non-menstrual RLS $(\mathrm{n}=14)$ & $p$ \\
\hline Serum iron $(\mu \mathrm{g} / \mathrm{dL})$ & $97.0 \pm 43.9$ & $85.1 \pm 38.2$ & 0.553 \\
Serum total iron binding capacity $(\mu \mathrm{g} / \mathrm{dL})$ & $311.3 \pm 20.2$ & $341.2 \pm 62.9$ & 0.141 \\
Serum iron saturation $(\%)$ & $31.2 \pm 13.6$ & $26.1 \pm 12.2$ & 0.421 \\
Serum ferritin $(\mu \mathrm{g} / \mathrm{mL})$ & $40.8[23.8-48.6]$ & $30.6[17.0-57.7]$ & 0.898 \\
Plasma hemoglobin $(\mathrm{g} / \mathrm{dL})$ & $13.8 \pm 1.2$ & $12.4 \pm 1.3$ & $0.044^{*}$ \\
\hline
\end{tabular}

Data are presented as mean \pm standard deviation or median [interquartile range]. ${ }^{*} p<0.05$. RLS: restless legs syndrome

inadequate timing of blood sample. In this study iron panel and hemoglobin levels were not taken during menstrual period. Comparing laboratory results from menstrual period to baseline may be more important than ferritin level itself. Patients with lower ferritin levels during menstrual period compared to baseline (even within reference value) are more likely to suffer from aggravated symptoms.

Another possibility is that iron depletion may take some time to develop symptom aggravation. In RLS patients, iron storage and release from endothelial cells of blood-brain bar- rier are altered. ${ }^{8}$ After iron-regulatory protein expressions are up- or down-regulated in response to systemic iron depletion, iron levels in brain tissue are decreased. Thus from iron depletion to symptom development, it may take several days or weeks. As iron supplement therapy takes several weeks for symptom improvement, it is reasonable assumption.

Moreover, iron depletion is not the only factor in developing RLS symptoms. Although RLS occurs 5 times more frequent in IDA than in general population, peripheral iron or hemoglobin levels are not different in IDA patients with RLS 
from those without RLS. ${ }^{9}$ This indicates that although iron deficiency plays crucial role, other factors such as genetic predispositions are required in developing RLS. For example, MEIS1 gene, which showed link to iron homeostasis in C. elegans, shows strong association with RLS.

This study had several limitations. First, limited number of premenopausal female RLS patients. Second, iron panel and hemoglobin levels were not taken during menstrual period. Comparing iron status during menstrual period to baseline may be helpful in future studies. Third, RLS symptom aggravations were answered on the basis of subjects' recall. Instead of cross-sectional study with questionnaire, longitudinal study with symptom diary can provide more precise description of symptom aggravations.

In this study we were not able to establish factors contributing to RLS symptom aggravation during menstrual cycle. But we confirmed that $30 \%$ of premenopausal female RLS patients suffer from symptom aggravation which is $40 \%$ more severe than non-menstrual period. Furthermore, sometimes it is so severe that patients need proper management during menstrual period. Further research is required to reveal mechanism of RLS symptom aggravation during menstrual period.

\section{Acknowledgments}

This work was supported by a National Research Foundation (NRF) grant funded by the Korean government (MSIP) (2017R1A2B2012280).

\section{Conflicts of Interest}

The authors have no potential conflicts of interest to disclose.

\section{ORCID iDs}

Sungeun Hwang https://orcid.org/0000-0003-0813-1315
Yong-Won Shin https://orcid.org/0000-0002-6781-7782

Ki-Young Jung https://orcid.org/0000-0001-5528-9081

\section{Author Contributions}

Conceptualization: Sungeun Hwang, Ki-Young Jung. Data curation: Sungeun Hwang, Yong-Won Shin. Formal analysis: Sungeun Hwang, Yong-Won Shin. Investigation: Sungeun Hwang. Methodology: Ki-Young Jung. Project administration: Sungeun Hwang, Ki-Young Jung. Resources: Ki-Young Jung. Writing_original draf: Sungeun Hwang. Writing-review \& editing: Yong-Won Shin, Ki-Young Jung.

\section{REFERENCES}

1. Allen RP, Picchietti DL, Garcia-Borreguero D, et al.; International Restless Legs Syndrome Study Group. Restless legs syndrome/WillisEkbom disease diagnostic criteria: updated International Restless Legs Syndrome Study Group (IRLSSG) consensus criteria-History, rationale, description, and significance. Sleep Med 2014;15:860-873.

2. Allen R. Dopamine and iron in the pathophysiology of restless legs syndrome (RLS). Sleep Med 2004;5:385-391.

3. Sun ER, Chen CA, Ho G, Earley CJ, Allen RP. Iron and the restless legs syndrome. Sleep 1998;21:371-377.

4. Avni T, Reich S, Lev N, Gafter-Gvili A. Iron supplementation for restless legs syndrome-A systematic review and meta-analysis. Eur J Intern Med 2019;63:34-41.

5. Allen RP, Walters AS, Montplaisir J, et al. Restless legs syndrome prevalence and impact: REST general population study. Arch Intern Med 2005; 165:1286-1292

6. Chen SJ, Shi L, Bao YP, et al. Prevalence of restless legs syndrome during pregnancy: a systematic review and meta-analysis. Sleep Med Rev 2018;40:43-54.

7. Ghorayeb I, Bioulac B, Scribans C, Tison F. Perceived severity of restless legs syndrome across the female life cycle. Sleep Med 2008;9:799-802.

8. Connor JR, Patton SM, Oexle K, Allen RP. Iron and restless legs syndrome: treatment, genetics and pathophysiology. Sleep Med 2017;31:61-70.

9. Allen RP, Auerbach S, Bahrain H, Auerbach M, Earley CJ. The prevalence and impact of restless legs syndrome on patients with iron deficiency anemia. Am J Hematol 2013;88:261-264. 